\title{
Transient conduction and convection heat transfer across a multi-layer floor subjected to multiple heat sources
}

\author{
Nuno Simões*, António Tadeu \\ Department of Civil Engineering, University of Coimbra, Polo II, 3030-290 Coimbra, Portugal
}

Received 5 May 2005; accepted 12 May 2005

\begin{abstract}
This paper analyses the transient heat transfer across multi-layer floors subjected to multiple heat sources. The formulation that is proposed to solve this problem uses analytical expressions handling both conduction and convection phenomena. The Green's functions (analytical expressions) for the layered formation are established by imposing the continuity of temperatures and heat fluxes at the medium interfaces between the various layers. The heat field inside a layer is obtained by adding the contribution of the direct heat incident field, generated as in an unbounded medium, to the heat produced by a set of virtual surface heat sources, that take into account the presence of the interfaces.

The technique used to deal with the unsteady state conditions consists of first computing the solution in the frequency domain and then applying (fast) inverse Fourier transforms into space-time.
\end{abstract}

(C) 2005 Elsevier Ltd. All rights reserved.

Keywords: Transient heat transfer; Conduction; Convection; Green's functions; Multi-layer systems; Multiple heat sources

\section{Introduction}

The interior comfort of a dwelling is a fundamental issue in building physics, and it depends on the building's envelope. In order to better understand the thermal performance of the construction elements used throughout the building envelope, it is increasingly important to develop more accurate models to evaluate it. Thermal behaviour depends largely on unsteady state conditions, and so the formulations for studying those systems should contemplate the transient heat phenomena. In general, multi-layer construction elements are used to ensure that all the functional requirements are met.

The heat transfer in multi-layer problems is a subject that has been quite widely researched. Several publications can be found in the literature: Özisik has written a

\footnotetext{
*Corresponding author. Tel.: + 351239797 191; fax: + 351239797190

E-mail address: nasimoes@dec.uc.pt (N. Simões).
}

book [1] including a review of one-dimensional composite media, referring to orthogonal expansions and Green's functions; Monte [2] analysed the transient heat conduction of multi-layer composite slabs, applying the method of separation of variables to the heat conduction partial differential equation. A frequency-domain regression method was developed by Wang and Chen [3] to compute the heat flow for a one-dimensional multilayer model. Many other earlier works describe transient heat transfer problems in composite layers, such as that by Haji-Sheikh et al. [4] and Sun and Wichman [5]. Haji-Sheikh et al. presented different types of Green's functions that are solutions of the heat conduction diffusion equation in multi-dimensional, multi-layer bodies for different boundary conditions, calculating eigenvalues, while Sun and Wichman used an eigenfunction expansion method to calculate the heat conduction in a one-dimensional three layer slab.

The study of the transient heat transfer can be handled either directly, in the time domain or in a transform space domain. In the time-marching 
approach the solution is assessed step by step at consecutive time intervals after an initially specified state has been assumed (see [6-8]). One approach widely used to overcome some of the disadvantages of the single-step time-marching schemes is the Laplace transform $[9,10]$. The Laplace transform employs a numerical transform inversion to calculate the physical variables in the real space, after the solution has been obtained for a sequence of values of the transform parameter. The major drawback of Laplace transforms is that accuracy is lost in the inversion process, which magnifies small truncation errors [11]. Davies [12] has published a work using a time domain analysis to study the transient heat flow in a multi-layer wall, while Chen et al. [13] uses Laplace transforms to calculate transient heat flow through multi-layer spherical structures. In this work, however, we propose a different approach, that needs the knowledge of Green's functions (analytical expressions) for a layered formation and the implementation of a frequency domain transform. This approach has been proposed and verified by the authors in a previous work (see [14]).

The search for Green's functions has been object of research over the years because these expressions are both useful by themselves and can be used as benchmark solutions [15]. These fundamental solutions can also be incorporated into numerical tools such as the Boundary Element Method (BEM) to solve more general problems (see [16]).

The Green's functions for calculating the transient heat transfer wave field in layered formations subjected to multiple heat sources are given below. The proposed fundamental solutions relate the heat field variables (heat fluxes or temperatures) along the domain caused by the energy generated by sources placed elsewhere in the media, in the presence of both conduction and convection phenomena. These expressions allow the heat field inside a layered medium to be computed, without the discretization of the interior domain. The problem is formulated in the frequency domain using time Fourier transforms.

This work extends the authors' earlier work on the definition of the conduction phenomenon within layered solid media (see [14,17]). The problem is now solved with the convection phenomenon being incorporated and considering multiple cylindrical heat sources. As in the previous work, the technique requires knowing the Green's functions for the case of a spatially sinusoidal, harmonic heat line source placed in an unbounded medium. The Green's functions for a layered formation are formulated as the sum of the heat source terms equal to those in the full-space and the surface terms required to satisfy the boundary conditions at the interfaces, i.e. continuity of temperatures and normal fluxes between layers. The total heat field is found by adding the heat source terms equal to those in the unbounded space to that set of surface terms, arising within each layer and at each interface.

This paper first formulates the three-dimensional problem and presents the Green's function for a sinusoidal heat line source applied in an unbounded medium. A brief description of the mathematical manipulation is given. Then, the Green's functions for a multi-layer formation, ensuring the continuity of temperatures and heat fluxes at the various interfaces, are established. Finally, the applicability of this formulation is illustrated by using it to analyse the heat propagation within a multi-layer floor subjected to several heat sources. Different simulations are studied, taking the convection phenomenon and the presence of an insulating layer into account.

\section{Three-dimensional problem formulation and Green's functions in an unbounded medium}

The transient convection-conduction heat transfer across homogeneous media, assuming null initial conditions, is expressed by the equation

$$
\begin{aligned}
& \left(\frac{\partial^{2}}{\partial x^{2}}+\frac{\partial^{2}}{\partial y^{2}}+\frac{\partial^{2}}{\partial z^{2}}\right) T-\frac{1}{K}\left(V_{x} \frac{\partial T}{\partial x}+V_{y} \frac{\partial T}{\partial y}+V_{z} \frac{\partial T}{\partial z}\right) T \\
& \quad=\frac{1}{K} \frac{\partial T}{\partial t}
\end{aligned}
$$

where $V_{x}, V_{y}$ and $V_{z}$ are the velocity components in the direction $x, y$ and $z$ respectively, $t$ is time, $T(t, x, y, z)$ is temperature, $K=k /(\rho c)$ is the thermal diffusivity, $k$ is the thermal conductivity, $\rho$ is the density and $c$ is the specific heat. It is presumed that the thermal properties and convection velocities along the $x, y$ and $z$ directions remain constant. Applying a Fourier transform in the time domain, one obtains an equation that differs from the Helmholtz equation by the inclusion of a convective term,

$$
\begin{aligned}
& \left(\left(\frac{\partial^{2}}{\partial x^{2}}+\frac{\partial^{2}}{\partial y^{2}}+\frac{\partial^{2}}{\partial z^{2}}\right)-\frac{1}{K}\left(V_{x} \frac{\partial}{\partial x}+V_{y} \frac{\partial}{\partial y}+V_{z} \frac{\partial}{\partial z}\right)\right. \\
& \left.+\left(\sqrt{\frac{-\mathrm{i} \omega}{K}}\right)^{2}\right) \hat{T}(\omega, x, y, z)=0,
\end{aligned}
$$

where $\mathrm{i}=\sqrt{-1}$ and $\omega$ is the frequency. The fundamental solution (Green's functions) of Eq. (2) for a heat point source in an unbounded medium, located at $(0,0,0)$, can be expressed as

$$
\begin{aligned}
\hat{T}_{f}(\omega, x, y, z)= & \frac{\mathrm{e}^{\frac{V_{x} x+V_{y} y+V_{z} z}{2 K}}}{2 k \sqrt{x^{2}+y^{2}+z^{2}}} \\
& \times \mathrm{e}^{-\mathrm{i} \sqrt{-\frac{V_{x}^{2}+V_{y}^{2}+V_{z}^{2}}{4 K^{2}}-\frac{\mathrm{i} \omega}{K}} \sqrt{x^{2}+y^{2}+z^{2}}} .
\end{aligned}
$$


The full three-dimensional problem can be mathematically manipulated if the geometry of the problem remains constant along one direction. Assuming that the geometry does not vary along the $z$ direction, the problem can be expressed as a summation of twodimensional solutions with different spatial wavenumbers $k_{z}$ [18] by means of the application of a Fourier transform along that direction. Considering that a spatially sinusoidal harmonic heat line source is applied at the point $(0,0)$ along the $z$ direction (see Fig. 1), subject to convection velocities $V_{x}, V_{y}$ and $V_{z}$, the application of a spatial Fourier transformation to

$\frac{\mathrm{e}^{-\mathrm{i} \sqrt{-\frac{V_{x}^{2}+V_{y}^{2}+V_{z}^{2}}{4 K^{2}}-\frac{\mathrm{i} \omega}{K}} \sqrt{x^{2}+y^{2}+z^{2}}}}{\sqrt{x^{2}+y^{2}+z^{2}}}$

along the $z$ direction, leads to

$$
\begin{aligned}
& \tilde{T}_{f}\left(\omega, x, y, k_{z}\right) \\
& \quad=\frac{-\mathrm{ie}\left(V_{x} x+V_{y} y+V_{z} z\right) / 2 K}{4 k} H_{0}\left(\sqrt{-\frac{V_{x}^{2}+V_{y}^{2}+V_{z}^{2}}{4 K^{2}}-\frac{\mathrm{i} \omega}{K}-\left(k_{z}\right)^{2}} r_{0}\right),
\end{aligned}
$$

where $H_{0}()$ are Hankel functions of the second kind and order 0 , and $r_{0}=\sqrt{\left(x-x_{0}\right)^{2}+\left(y-y_{0}\right)^{2}}$.

The full three-dimensional solution is then retrieved by means of an inverse Fourier transform along the $k_{z}$ domain. This inverse Fourier transformation can be expressed as a discrete summation if we assume the existence of an infinite set of virtual sources, equally spaced at $L_{z}$, along $z$, which enables the solution to be obtained by solving a limited number of two-dimensional problems,

$\hat{T}(\omega, x, y, z)=\frac{2 \pi}{L} \sum_{m=-M}^{M} \tilde{T}\left(\omega, x, y, k_{z m}\right) \mathrm{e}^{-\mathrm{i} k_{z m} z}$

with $k_{z m}$ being the axial wavenumber given by $k_{z m}=\left(2 \pi / L_{z}\right) m$. The distance $L_{z}$ is chosen so as to prevent spatial contamination from the virtual sources [19].

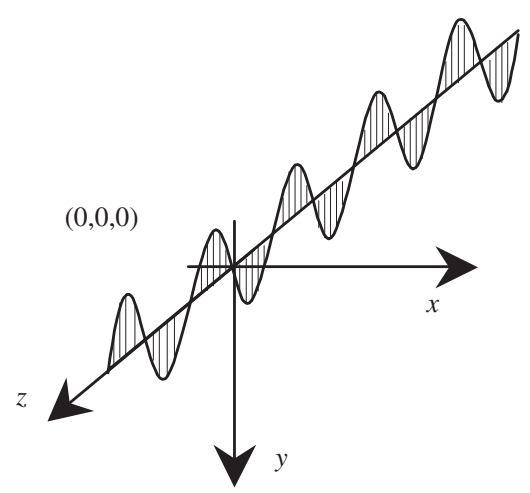

Fig. 1. Spatially harmonic varying line load.
The application of a spatial Fourier transformation along the $z$ direction in Eq. (2) leads to the following equation:

$$
\begin{aligned}
& \left(\left(\frac{\partial^{2}}{\partial x^{2}}+\frac{\partial^{2}}{\partial y^{2}}\right)-\frac{1}{K}\left(V_{x} \frac{\partial}{\partial x}+V_{y} \frac{\partial}{\partial y}\right)\right. \\
& \left.+\left(\sqrt{\frac{-\mathrm{i} \omega}{K}-\left(k_{z}\right)^{2}}\right)^{2}\right) \tilde{T}\left(\omega, x, y, k_{z}\right)=0
\end{aligned}
$$

when $V_{z}=0$. The fundamental solution of this equation is given by Eq. (5) ascribing $V_{z}=0$.

Note that the problem can be approached as a continuous superposition of heat plane effects: Eq. (5) may be substituted by the expression,

$$
\begin{aligned}
\tilde{T}_{f}\left(\omega, x, y, k_{z}\right)= & \frac{-\mathrm{ie}^{\left(V_{x} x+V_{y} y+V_{z} z\right) / 2 K}}{4 \pi k} \int_{-\infty}^{+\infty} \\
& \times\left(\frac{\mathrm{e}^{-\mathrm{i}\left|y-y_{0}\right|}}{v}\right) \mathrm{e}^{-\mathrm{i} k_{x}\left(x-x_{0}\right)} \mathrm{d} k_{x},
\end{aligned}
$$

where

$v=\sqrt{-\left(\left(V_{x}^{2}+V_{y}^{2}+V_{z}^{2}\right) / 2 K\right)-(\mathrm{i} \omega / K)-\left(k_{z}\right)^{2}-k_{x}^{2}}$

with $(\operatorname{Im}(v) \leqslant 0)$, and the integration is performed with respect to the horizontal wave number $\left(k_{x}\right)$ along the $x$ direction.

The integral in the above equation can be transformed into a summation if an infinite number of such sources are distributed along the $x$ direction, at equal intervals $L_{x}$. Each individual heat source is simulated, ensuring that the distance separating it from its neighbours is large enough to prevent the virtual loads from contaminating the heat responses; the simulation of a system using multiple heat sources is obtained by ascribing the exact distance between these virtual loads. Therefore, Eq. (8) can then be expressed as

$\tilde{T}_{f}\left(\omega, x, y, k_{z}\right)=\frac{-\mathrm{ie}^{\left(V_{x} x+V_{y} y+V_{z} z\right) / 2 K}}{4 k} E_{0} \sum_{n=-\infty}^{n=+\infty}\left(\frac{E}{v_{n}}\right) E_{d}$,

where $\quad E_{0}=\frac{-\mathrm{i}}{2 k L_{x}}, \quad E=\mathrm{e}^{-\mathrm{i} v_{n}\left|y-y_{0}\right|}, \quad E_{d}=\mathrm{e}^{-\mathrm{i} k_{x n}\left(x-x_{0}\right)}$, $v_{n}=\sqrt{-\left(\left(V_{x}^{2}+V_{y}^{2}+V_{z}^{2}\right) / 4 K^{2}\right)-(\mathrm{i} \omega / K)-\left(k_{z}\right)^{2}-k_{x n}^{2}}$ with $\left(\operatorname{Im}\left(v_{n}\right) \leqslant 0\right), k_{x n}=\left(2 \pi / L_{x}\right) n$, which can in turn be approximated by a finite sum of equations $(N)$. Note that $k_{z}=0$ corresponds to the two-dimensional case.

After computing the heat field in the frequency domain, the spatial-temporal response is calculated by applying numerical inverse fast Fourier transforms in $k_{z}, k_{x}$ and in the frequency domain $(\omega)$. The time variation of the source can be easily defined or changed, which allows the modelling of the appropriate heat power conditions. To prevent interference from aliasing phenomena, the computations are performed using 
complex frequencies with a small imaginary part of the form $\omega_{c}=\omega-\mathrm{i} \eta$ (with $\eta=0.7 \Delta \omega$, and $\Delta \omega$ being the frequency step). In the time domain, this effect is removed by rescaling the response with an exponential window of the form $\mathrm{e}^{\eta t}$.

\subsection{Verification of the solution}

The exact solution of the transient conductionconvection heat transfer for an unbounded medium subjected to a three, two or one-dimensional unit heat source is well known (see [20]). Assuming that a unit heat source, applied at time $t=t_{0}$, is placed at point $(0,0,0)$, the exact time domain solution of the temperature at $(x, y, z)$, considering both conduction and convection phenomena, is given by

$$
\begin{aligned}
& T(t, x, y, z)=\frac{\mathrm{e}^{\left(-\left(-\tau V_{x}+x\right)^{2}-\left(-\tau V_{y}+y\right)^{2}-\left(-\tau V_{z}+z\right)^{2}\right) / 4 K \tau}}{\rho c(4 \pi K \tau)^{d / 2}}, \\
& \quad \text { if } t>t_{0}
\end{aligned}
$$

where $\tau=t-t_{0}$; the parameter $d$ can be 3,2 or 1 depending on whether the problem is three, two or onedimensional, respectively.

The performance of the formulation stated above is evaluated in the calculation of the heat field in a homogeneous unbounded medium with thermal properties that allow $k=1.4 \mathrm{~W} \mathrm{~m}^{-1}{ }^{\circ} \mathrm{C}^{-1}, \quad c=$ $880.0 \mathrm{~J} \mathrm{Kg}^{-1}{ }^{\circ} \mathrm{C}^{-1}$ and $\rho=2300 \mathrm{Kg} \mathrm{m}^{-3}$. The convection velocities applied in the $x, y$ and $z$ direction were equal to $1 \times 10^{-6} \mathrm{~m} \mathrm{~s}^{-1}$. If a cylindrical $(d=2)$ unit heat source placed at $\left(x_{0}=0.0 \mathrm{~m}, y_{0}=0.0 \mathrm{~m}, z_{0}=0.0 \mathrm{~m}\right)$ is excited at $t=277.8 \mathrm{~h}$, the responses at different times $(350,450,550$ and $650 \mathrm{~h})$ calculated along a line of 40 receivers placed from $(x=-1.5, y=0.35 z=0.0)$ to $(x=1.5, y=0.35, z=0.0)$, are those given in Fig. 2. In this figure, the solid lines represent the exact time solution given by Eq. (10) while the marks show the response obtained using the proposed Green's functions. A good agreement was achieved between these two solutions. As we have assigned a convection velocity in the $x$ direction, it should be noted that the temperature response along the line of receivers is not symmetrical.

The calculations were first performed in the frequency domain, in the frequency range $\left[0,1024 \times 10^{-7} \mathrm{~Hz}\right.$, with an increment of $\Delta \omega=10^{-7} \mathrm{~Hz}$, which defines a time window of $2777.8 \mathrm{~h}$.

\section{Green's functions in a layered formation}

This section establishes the Green's functions for a layered formation, using the continuity of temperatures and heat fluxes as boundary conditions at the interfaces between layers.

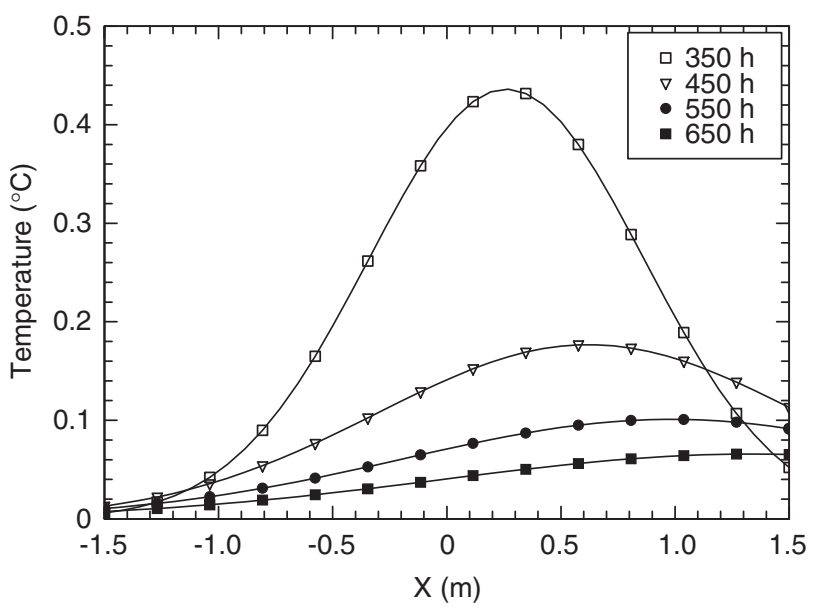

Fig. 2. Temperature distribution along a line of 40 receivers placed in an unbounded medium, at different times $(350,450,550$ and $650 \mathrm{~h})$ in the presence of a cylindrical $(d=2)$ unit heat source.

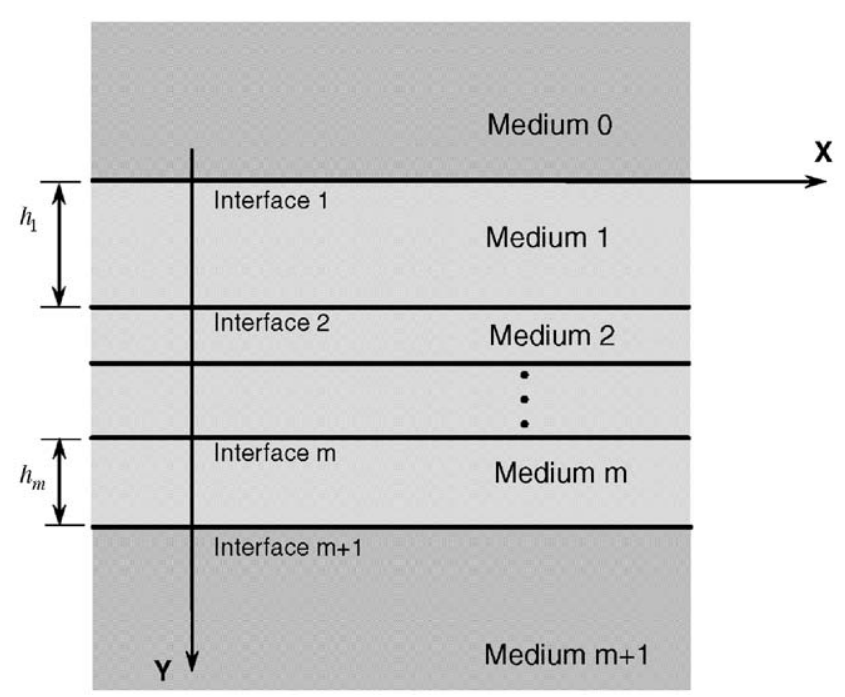

Fig. 3. Geometry of the problem for a multi-layer system bounded by two semi-infinite media (media 0 and $m+1$ ).

Consider a set of $m$ homogeneous plane layers of infinite extent bounded by two flat, semi-infinite, media (see Fig. 3). The top semi-infinite medium has been called medium 0 , while the bottom semi-infinite medium is taken to be medium $m+1$. Different material thermal properties, vertical convection velocities and thicknesses can be ascribed to each layer. These convection velocities are perpendicular to the layer's plane. Note that convection phenomenon is modelled assuming that its origin coincides with the heat source. Assuming that the multi-layer is subjected to a spatially sinusoidal heat source placed in medium 1, the heat field at any position is computed taking into account both the surface heat terms generated at each interface and the contribution of the heat source term (incident field). 
For the layer $j$, the heat surface terms on the upper and lower interfaces can be expressed as

$\tilde{T}_{j 1}\left(\omega, x, y, k_{z}\right)=E_{0 j} \mathrm{e}^{V_{y j}\left(y-y_{0}\right) / 2 K_{j}} \sum_{n=-\infty}^{n=+\infty}\left(\frac{E_{j 1}}{v_{n j}} A_{n j}^{t}\right) E_{d}$,

$\tilde{T}_{j 2}\left(\omega, x, y, k_{z}\right)=E_{0 j} \mathrm{e}^{V_{y j}\left(y-y_{0}\right) / 2 K_{j}} \sum_{n=-\infty}^{n=+\infty}\left(\frac{E_{j 2}}{v_{n j}} A_{n j}^{b}\right) E_{d}$,

where $E_{0 j}=-\mathrm{i} / 2 k_{j} L_{x}, \quad E_{j 1}=\mathrm{e}^{-\mathrm{i} v_{n j}\left|y-\sum_{l=1}^{j-1} h_{l}\right|}, \quad E_{j 2}=$ $\mathrm{e}^{-\mathrm{i} v_{n j}\left|y-\sum_{l=1}^{j} h_{l}\right|}$.

$v_{n j}=\sqrt{-\left(V_{y} / 2 K_{j}\right)^{2}+-\mathrm{i} \omega / K_{j}-k_{z}^{2}-k_{x n}^{2}}$, with $\operatorname{Im}\left(v_{n j}\right) \leqslant$ 0 and $h_{l}$ is the thickness of the layer $l$. Meanwhile, $K_{j}=$ $k_{j} /\left(\rho_{j} c_{j}\right)$ is the thermal diffusivity in the medium $j\left(k_{j}, \rho_{j}\right.$ and $c_{j}$ represent the thermal conductivity, the density and the specific heat of the material in the medium, $j$, respectively). The heat surface terms produced at interfaces 1 and $m+1$, governing the heat that propagates through the top and bottom semi-infinite media, are respectively expressed by

$\tilde{T}_{02}\left(\omega, x, y, k_{z}\right)=E_{00} \mathrm{e}^{V_{y 0}\left(y-y_{0}\right) / 2 K_{0}} \sum_{n=-\infty}^{n=+\infty}\left(\frac{E_{01}}{v_{n 0}} A_{n 0}^{b}\right) E_{d}$,

$$
\begin{aligned}
\tilde{T}_{(m+1) 1}\left(\omega, x, y, k_{z}\right)= & E_{0(m+1)} \mathrm{e}^{V_{y(m+1)}\left(y-y_{0}\right) / 2 K_{(m+1)}} \\
& \times \sum_{n=-\infty}^{n=+\infty}\left(\frac{E_{(m+1) 2}}{v_{n(m+1)}} A_{n(m+1)}^{t}\right) E_{d} .
\end{aligned}
$$

The system of equations is assembled, imposing the continuity of temperatures and heat fluxes along the $m+1$ interfaces between layers. $2(m+1)$ equations are achieved, taking into account the contribution of the surface terms and the involvement of the incident field. All the terms are organized according to the form $\underline{F} \underline{a}=\underline{b}$ where

$c_{1 j}=\left[V_{y j} / 2 K_{j}+\mathrm{i} v_{n j}\right]$,

$c_{2 j}=\left[V_{y j} / 2 K_{j}-\mathrm{i} v_{n j}\right]$,

$c_{3 j}=\frac{\mathrm{e}^{V_{y j}\left(\sum_{l=1}^{j} h_{l}-y_{0}\right) / 2 K_{j}}}{v_{n j}}$,

$c_{4 j}=\frac{\mathrm{e}^{V_{y j}\left(\sum_{l=1}^{j-1} h_{l}-y_{0}\right) / 2 K_{j}}}{v_{n j}}$

and $c_{5 j}=\mathrm{e}^{-\mathrm{i} v_{n j} h_{j}}$.

The resolution of the system of equations allows the unknown amplitudes of the surface terms in each interface $\left(A_{n 0}^{b}, A_{n 1}^{t}, A_{n 1}^{b}, \ldots A_{n m}^{t}, A_{n m}^{b}, A_{n(m+1)}^{t}\right)$ to be computed. The heat field for each layer formation is then obtained by adding these surface terms (generated heat field) to the contribution of the incident field, leading to the following equations:

Top semi-infinite medium (medium 0 )

$$
\tilde{T}\left(\omega, x, y, k_{z}\right)=E_{00} \mathrm{e}^{V_{y 0}\left(y-y_{0}\right) / 2 K_{0}} \sum_{n=-\infty}^{n=+\infty}\left(\frac{E_{01}}{v_{n 0}} A_{n 0}^{b}\right) E_{d},
$$

if $y<0$,

medium 1 (source position)

$$
\begin{aligned}
\tilde{T}\left(\omega, x, y, k_{z}\right)= & \frac{-\mathrm{i}}{4 k_{1}} \mathrm{e}^{V_{y 1}\left(y-y_{0}\right) / 2 K_{1}} H_{0}\left(K_{1} r_{0}\right) \\
& +E_{01} \mathrm{e}^{V_{y 1}\left(y-y_{0}\right) / 2 K_{1}} \\
& \times \sum_{n=-\infty}^{n=+\infty}\left(\frac{E_{11}}{v_{n 1}} A_{n 1}^{t}+\frac{E_{12}}{v_{n 1}} A_{n 1}^{b}\right) E_{d}, \\
& \text { if } 0<y<h_{1},
\end{aligned}
$$

$$
\left[\begin{array}{ccccccc}
c_{10} c_{30} & -c_{21} c_{41} & -c_{11} c_{41} c_{51} & \cdots & 0 & 0 & 0 \\
\frac{c_{30}}{k_{0}} & -\frac{c_{41}}{k_{1}} & -\frac{c_{41} c_{51}}{k_{1}} & \cdots & 0 & 0 & 0 \\
0 & c_{21} c_{31} c_{51} & c_{11} c_{31} & \cdots & 0 & 0 & 0 \\
0 & \frac{c_{31} c_{51}}{k_{1}} & \frac{c_{31}}{k_{1}} & \cdots & 0 & 0 & 0 \\
\cdots & \cdots & \cdots & \cdots & \cdots & \cdots & \cdots \\
0 & 0 & 0 & \cdots & -c_{2 m} c_{4 m} & -c_{1 m} c_{4 m} c_{5 m} & 0 \\
0 & 0 & 0 & \cdots & -\frac{c_{4 m}}{k_{m}} & -\frac{c_{41} c_{5 m}}{k_{m}} & 0 \\
0 & 0 & 0 & \cdots & c_{2 m} c_{3 m} c_{5 m} & c_{1 m} c_{3 m} & -c_{2(m+1)} c_{4(m+1)} \\
0 & 0 & 0 & \cdots & \frac{c_{3 m} c_{5 m}}{k_{m}} & \frac{c_{3 m}}{k_{m}} & -\frac{c_{4(m+1)}}{k_{(m+1)}}
\end{array}\right]\left[\begin{array}{c}
A_{n 0}^{b} \\
A_{n 1}^{t} \\
A_{n 1}^{b} \\
\cdots \\
A_{n m}^{t} \\
A_{n m}^{b} \\
A_{n(m+1)}^{t}
\end{array}\right]=\left[\begin{array}{c}
c_{11} c_{41} \mathrm{e}^{-\mathrm{i} v_{n 1} y_{0}} \\
\frac{c_{41}}{k_{1}} \mathrm{e}^{-\mathrm{i} v_{n 1} y_{0}} \\
-c_{21} c_{31} \mathrm{e}^{-\mathrm{i} v_{n 1}\left|h_{1}-y_{0}\right|} \\
-\frac{c_{31}}{k_{1}} \mathrm{e}^{-\mathrm{i} v_{n 1}\left|h_{1}-y_{0}\right|} \\
\ldots \\
0 \\
0 \\
0 \\
0 \\
0
\end{array}\right]
$$


medium $j(j \neq 1)$

$$
\begin{aligned}
\tilde{T}\left(\omega, x, y, k_{z}\right)= & E_{0 j} \mathrm{e}^{V_{y j}\left(y-y_{0}\right) / 2 K_{j}} \\
& \times \sum_{n=-\infty}^{n=+\infty}\left(\frac{E_{j 1}}{v_{n j}} A_{n j}^{t}+\frac{E_{j 2}}{v_{n j}} A_{n j}^{b}\right) E_{d}, \\
& \text { if } \sum_{l=1}^{j-1} h_{l}<y<\sum_{l=1}^{j} h_{l},
\end{aligned}
$$

bottom semi-infinite medium $($ medium $m+1)$

$$
\begin{aligned}
\tilde{T}_{(m+1) 2}\left(\omega, x, y, k_{z}\right)= & E_{0(m+1)} \mathrm{e}^{V_{y(m+1)}\left(y-y_{0}\right) / 2 K_{m+1}} \\
& \times \sum_{n=-\infty}^{n=+\infty}\left(\frac{E_{(m+1) 2}}{v_{n(m+1)}} A_{n(m+1)}^{t}\right) E_{d}, \\
& \text { if } y>\sum_{l=1}^{j} h_{l} .
\end{aligned}
$$

Note that if the heat source is placed in another layer, the matrix $\underline{\underline{F}}$ remains the same, while the independent terms of $\underline{b}$ are different. However, as the equations can be easily manipulated to consider another position for the source, they are not included here.

The responses in the time domain are calculated by means of a numerical inverse fast Fourier transform in $k_{z}, k_{x}$ and $\omega$, as explained above.

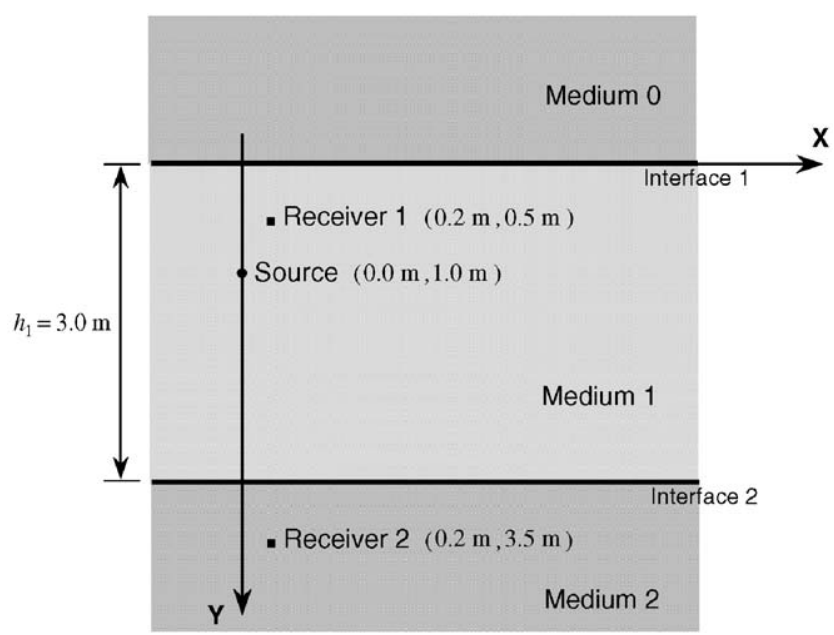

Fig. 4. Geometry of the problem for a layer bounded by two semiinfinite media.

\section{Green's functions verification}

The accuracy of this layered problem formulation was verified by comparing its results with those obtained using a BEM. This technique entails high computational costs, as is well known, since boundary meshing is required. To better simulate the layer interfaces, the boundary is discretized with a large number of elements distributed along as much of the surface as necessary. The methodology that is adopted to delimit the interfaces' discretization involves introducing an imaginary part to the frequencies, $\omega_{c}=\omega-i \eta$ (with $\eta=0.7 \Delta \omega)$, which introduces damping.
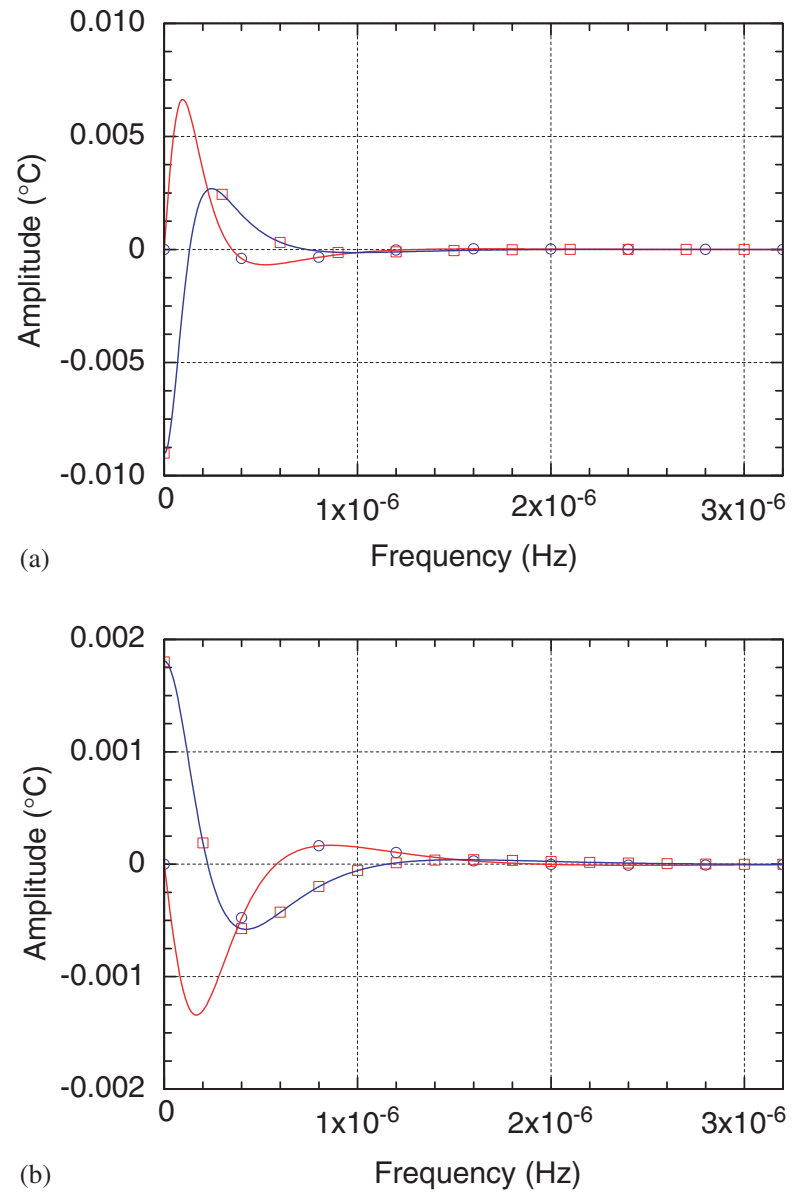

Fig. 5. Real and imaginary parts of the responses for a layer bounded by two semi-infinite media: (a) Receiver 1 and (b) Receiver 2 .

Table 1

Material's thermal properties for verification of the Green's functions

\begin{tabular}{llll}
\hline & Intermediate layer (medium 1) & Bottom medium (medium 2) & Top medium (medium 0) \\
\hline Thermal conductivity $\left(\mathrm{W} \mathrm{m}^{-1}{ }^{\circ} \mathrm{C}^{-1}\right)$ & $k_{1}=1.4$ & $k_{2}=63.9$ & $k_{0}=63.9$ \\
Density $\left(\mathrm{kg} \mathrm{m}^{-3}\right)$ & $\rho_{1}=2300.0$ & $\rho_{2}=7832.0$ & $\rho_{0}=7832.0$ \\
Specific heat $\left(\mathrm{J} \mathrm{kg}^{-1}{ }^{\circ} \mathrm{C}^{-1}\right)$ & $c_{1}=880.0$ & $c_{2}=434.0$ & $c_{0}=434.0$
\end{tabular}


The verification procedure is performed calculating the temperature field generated by a heat source placed in a flat layer, $3.0 \mathrm{~m}$ thick, bounded by two semi-infinite media (see Fig. 4). The convection velocities, in the $y$ direction, applied to the three media were $5 \times 10^{-7}, 8 \times$ $10^{-7}$ and $1 \times 10^{-6} \mathrm{~m} \mathrm{~s}^{-1}$ for the top medium, intermediate layer and bottom medium, respectively. The material's thermal properties used are presented in Table 1.

The calculations have been computed for 32 frequencies starting from $0 \mathrm{~Hz}$, assuming a frequency increment of $\Delta \omega=10^{-7} \mathrm{~Hz}$ and a single value of $k_{z}$ equal to $0.4 \mathrm{rad} \mathrm{m}^{-1}$. The responses were calculated for two receivers placed in two different media when a heat point source was located at $(x=0.0 \mathrm{~m}, y=1.0 \mathrm{~m})$. Fig. 5 displays the real and imaginary parts of the response at receiver $1(x=0.2 \mathrm{~m}, y=0.5 \mathrm{~m})$ and receiver $2(x=0.2 \mathrm{~m}, y=3.5 \mathrm{~m})$, placed in mediums 1 and 2 , respectively. The imaginary part of the frequency was set to $\eta=0.7 \Delta \omega$. The solid lines represent the analytical responses, while the marked points correspond to the

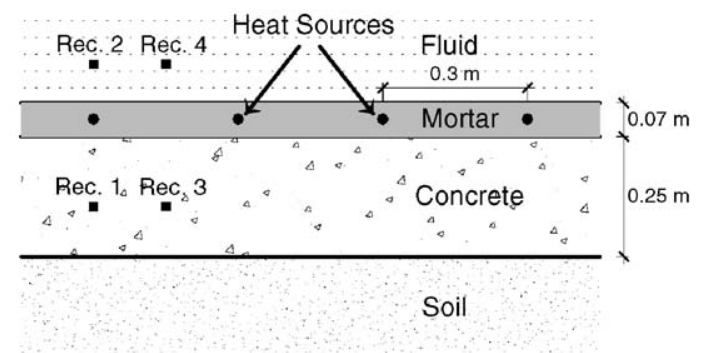

(a)

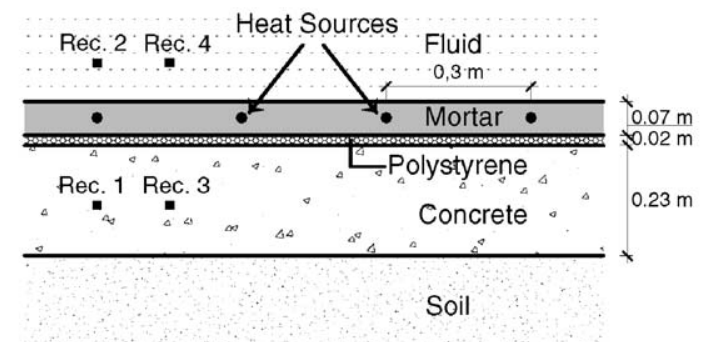

(c)
BEM solution. The square and the round marks designate the real and imaginary parts of the responses, respectively. As can be seen, these two solutions seem to be in very close agreement, and equally good results were obtained from tests in which heat sources and receivers were situated at different points.

\section{Applications}

The applicability of the formulation presented above is now illustrated. The heat propagation through a floor ( $0.32 \mathrm{~m}$ thick), composed by several flat infinite layers bounded by two semi-infinite media, is computed for different cases. The incorporation of the convection phenomenon and the presence of an insulating layer and its position are analysed.

The top semi-infinite layer of the multi-layer system is assumed to be a fluid (water or air), allowing a vertical convection velocity of $5 \mathrm{e}^{-6} \mathrm{~ms}^{-1}$, while the bottom semi-infinite layer is considered to be soil. The upper

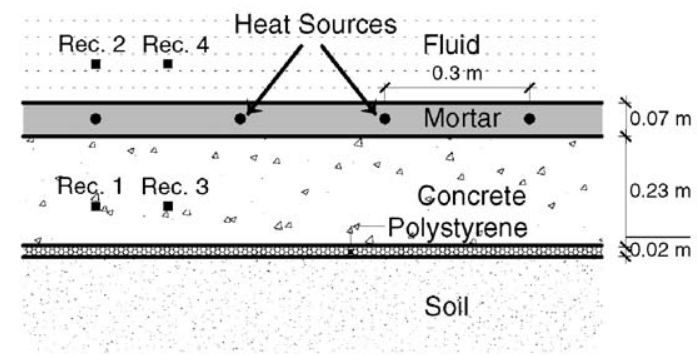

(b)

(d)

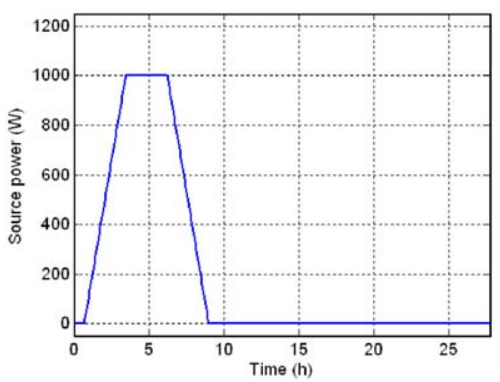

Fig. 6. Schematic illustration of the problem's geometries: Model 1 (a), Model 2 (b) and Model 3 (c). Time evolution of the power emitted by the heat source (d).

Table 2

Materials' thermal properties of the application examples

\begin{tabular}{|c|c|c|c|}
\hline & Thermal conductivity, $k\left(\mathrm{~W} \mathrm{~m}^{-1}{ }^{\circ} \mathrm{C}^{-1}\right)$ & Density, $\rho\left(\mathrm{kg} \mathrm{m}^{-3}\right)$ & Specific heat, $c\left(\mathrm{~J} \mathrm{~kg}^{-1}{ }^{\circ} \mathrm{C}^{-1}\right)$ \\
\hline Air & 0.0267 & 1.177 & 1005.0 \\
\hline Water & 0.606 & 998.0 & 4181.0 \\
\hline Cement mortar & 0.72 & 1860.0 & 780.0 \\
\hline Concrete & 1.4 & 2300.0 & 880.0 \\
\hline Polystyrene & 0.027 & 55.0 & 1210.0 \\
\hline Soil & 0.52 & 2050.0 & 1840.0 \\
\hline
\end{tabular}


solid layer, $0.07 \mathrm{~m}$ thick, is made of cement mortar. The remaining layers, placed between the cement mortar layer and the soil, may be modelled as concrete and thermal insulating material. Three different geometries are modelled: Model 1 only considers a concrete layer with a thickness of $0.25 \mathrm{~m}$; in Model 2 an insulating layer (extruded polystyrene), $0.02 \mathrm{~m}$ thick, is located below the concrete layer, which is $0.23 \mathrm{~m}$ thick, while in Model 3 the insulating material is positioned above the concrete layer. The models' geometries are shown in
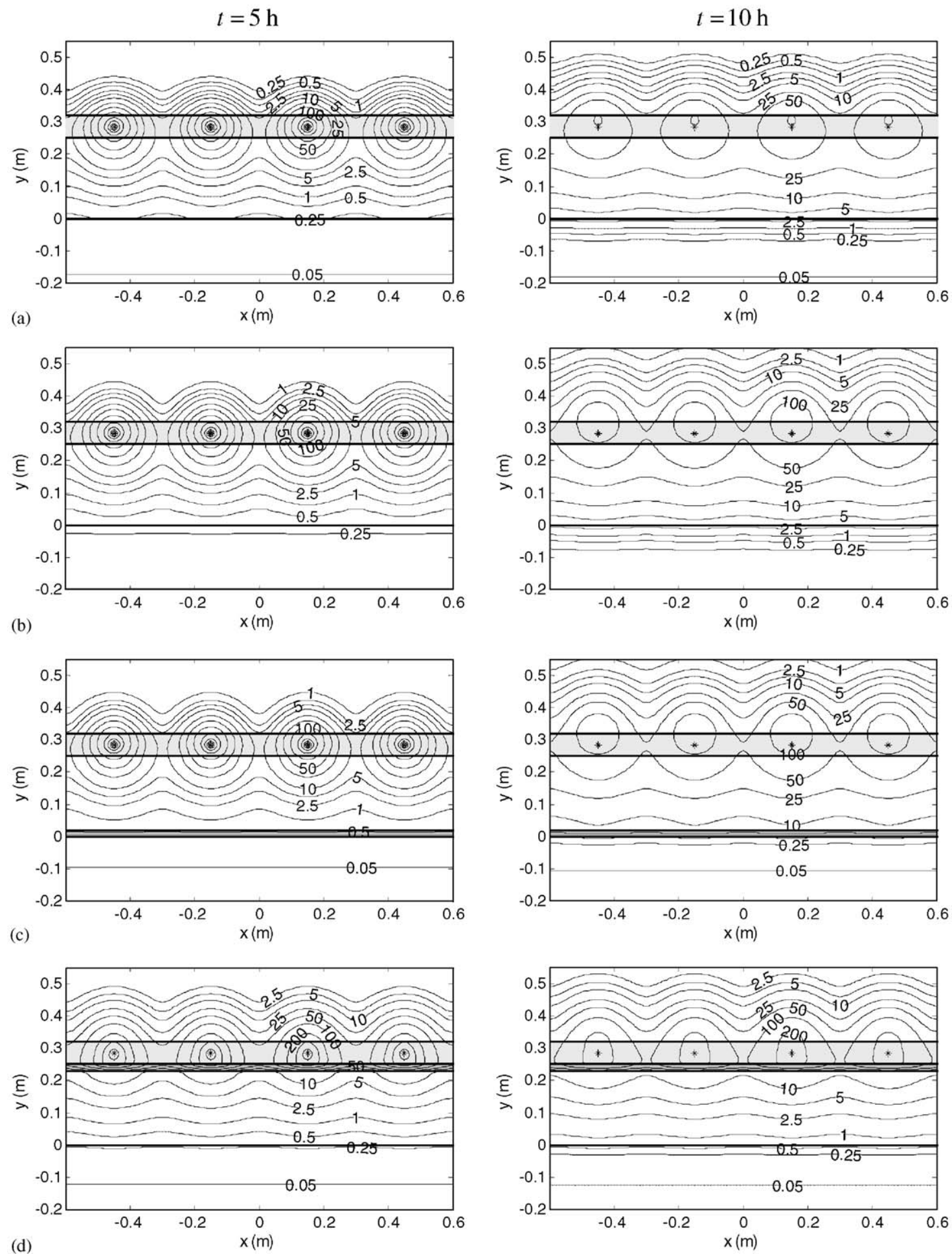

Fig. 7. Temperature distribution across the multi-layer floor at $t=5$ and $10 \mathrm{~h}$. (a) Model 1 , when the fluid is water without convection velocity; (b) Model 1, when the fluid is water with convection velocity; (c) Model 2, when the fluid is water with convection velocity and (d) Model 3, when the fluid is water with convection velocity. 
Fig. 6, while the materials' thermal properties are listed in Table 2.

The layered floors are subjected to the heat generated by several spatially sinusoidal harmonic heat line sources placed inside the cement mortar layer at equal distances of $0.3 \mathrm{~m}$ apart $\left(L_{x}=0.3 \mathrm{~m}\right)$. These sources start emitting energy at $t \approx 0.76 \mathrm{~h}$, as shown in Fig. 6(d). Their power is increased linearly from 0.0 to $1000.0 \mathrm{~W}$, reaching maximum power at $t \approx 3.46 \mathrm{~h}$. This value is maintained for a period of $t \approx 2.72 \mathrm{~h}$. The power is then reduced linearly to $0.0 \mathrm{~W}$, which occurs at $t \approx 8.89 \mathrm{~h}$. The heat fields are computed over a fine grid of receivers in the frequency range of $\left[0.0,128.0 \mathrm{e}^{-5} \mathrm{~Hz}\right]$, with a frequency increment of $1.0 \mathrm{e}^{-5} \mathrm{~Hz}$, which establishes a time period of $27.78 \mathrm{~h}$. The calculations have been performed for a grid of receivers, placed at $0.01 \mathrm{~m}$ intervals along the $x$ direction, and at $0.08 \mathrm{~m}$ along the $y$ direction. In order to study the performance of each constructive system, the temperatures for the three models have been plotted across the full domain at different times (snapshots) and also at specific receivers for the complete time window.

Fig. 7 shows a sequence of snapshots of the results obtained at different times, $t=5$ and $t=10 \mathrm{~h}$, when the fluid medium is filled with water. These figures display the temperature field as isothermal contour plots. Notice that all the receivers register a null temperature at the beginning of the process, to conform with the initial conditions defined for the present problem.

Fig. 7(a) and (b) give the results for Model 1, when the fluid is assumed to be water. In subheading (a) the convection phenomenon through the water medium is not modelled, while in Fig. 7(b) a vertical convection velocity of $5 \mathrm{e}^{-6} \mathrm{~m} \mathrm{~s}^{-1}$ is ascribed to the water medium. These results show perceptible amplitude differences between the two cases. In both cases it can be seen that the energy spreads faster through the concrete layer, which is in accordance with its higher diffusivity $(K)$. At time $t=5 \mathrm{~h}$ the contour configuration between the two cases is very similar, but higher temperatures can be already found along the top semi-infinite medium in the presence of the convection phenomenon, since the heat propagates more rapidly. At time $t=10 \mathrm{~h}$ the source power is no longer emitting energy, but the energy continues to propagate through the full domain of receivers. The temperature increases for the receivers located further away from the source, while a temperature fall is registered at the receivers closer to the heat sources. This behaviour occurs while the energy is achieving equilibrium throughout the full domain. The heat field that can be observed immediately below the concrete layer is very similar for the two situations (Fig. 7(a) and (b) at $t=10 \mathrm{~h}$ ).

The responses presented in Fig. 7(c) and (d) corresponding to Models 2 and 3, respectively, assume both
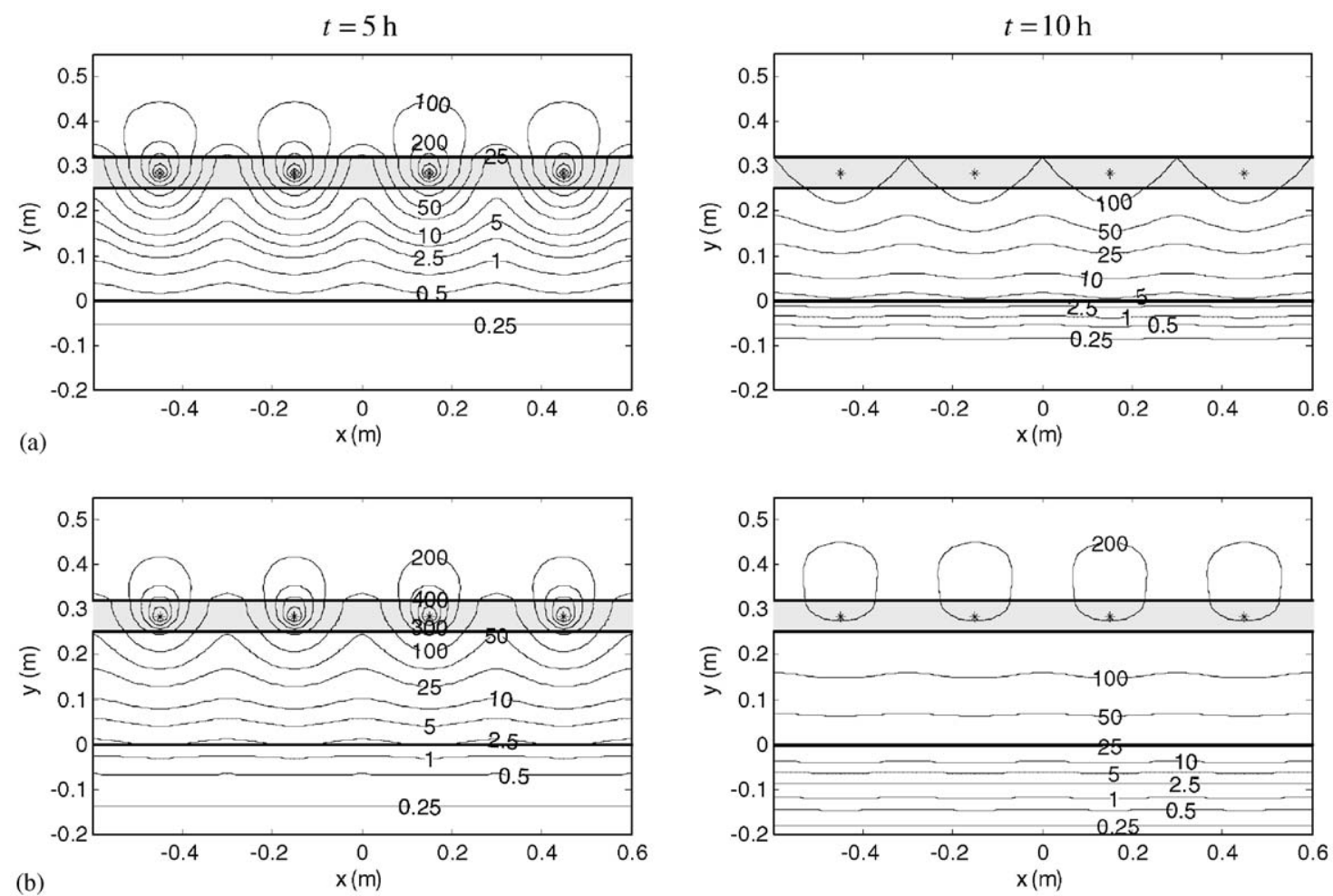

Fig. 8. Temperature distribution across the multi-layer floor, at $t=5$ and $10 \mathrm{~h}$, for Model 1 , when the fluid is air: (a) conduction phenomenon and (b) conduction and convection phenomena. 
conduction and convection phenomena. These models have been set to describe the heat field changes when there is an insulating layer. The introduction of an insulating layer, $0.02 \mathrm{~m}$ thick, between the soil and the concrete insulating (Model 2) layer allows a slight temperature rise in the concrete layer and, as expected, lower temperatures in the soil medium, as shown in Fig. 7(c). A considerable thermal gradient is noted across the thermal insulating layer, due to the low thermal conductivity of this material.

Fig. 7(d) displays the temperature distribution (isothermals) for the case where the insulating layer is located immediately below the source-containing layer. This case exhibits higher temperatures throughout the water medium, since the energy is not spreading so easily to the concrete layer as in the other cases. A large thermal gradient is clearly visible between the top and bottom interfaces of the insulating layer. Note that the concrete layer is heat-protected by the presence of the insulating layer. For example, at time $t=10 \mathrm{~h}$, close to the sources, temperatures of 300 and $50{ }^{\circ} \mathrm{C}$ are registered at the top and bottom interfaces, respectively. This reveals the importance of the insulating layer's position, which determines the quantity of energy dissipated wastefully. If the convection phenomenon for Models 2 and 3 is not taken into account (these plots are not included here), the minimum temperature registered in the water medium of Models 2 and 3 , at $t=5 \mathrm{~h}$, was $0.25^{\circ} \mathrm{C}$, instead of 1 and $2.5^{\circ} \mathrm{C}$, registered when the convection is modelled, as shown in Fig. 7(c) and (d).

Fig. 8 presents the snapshots displaying the temperature field for Model 1 when the fluid is air. Comparing
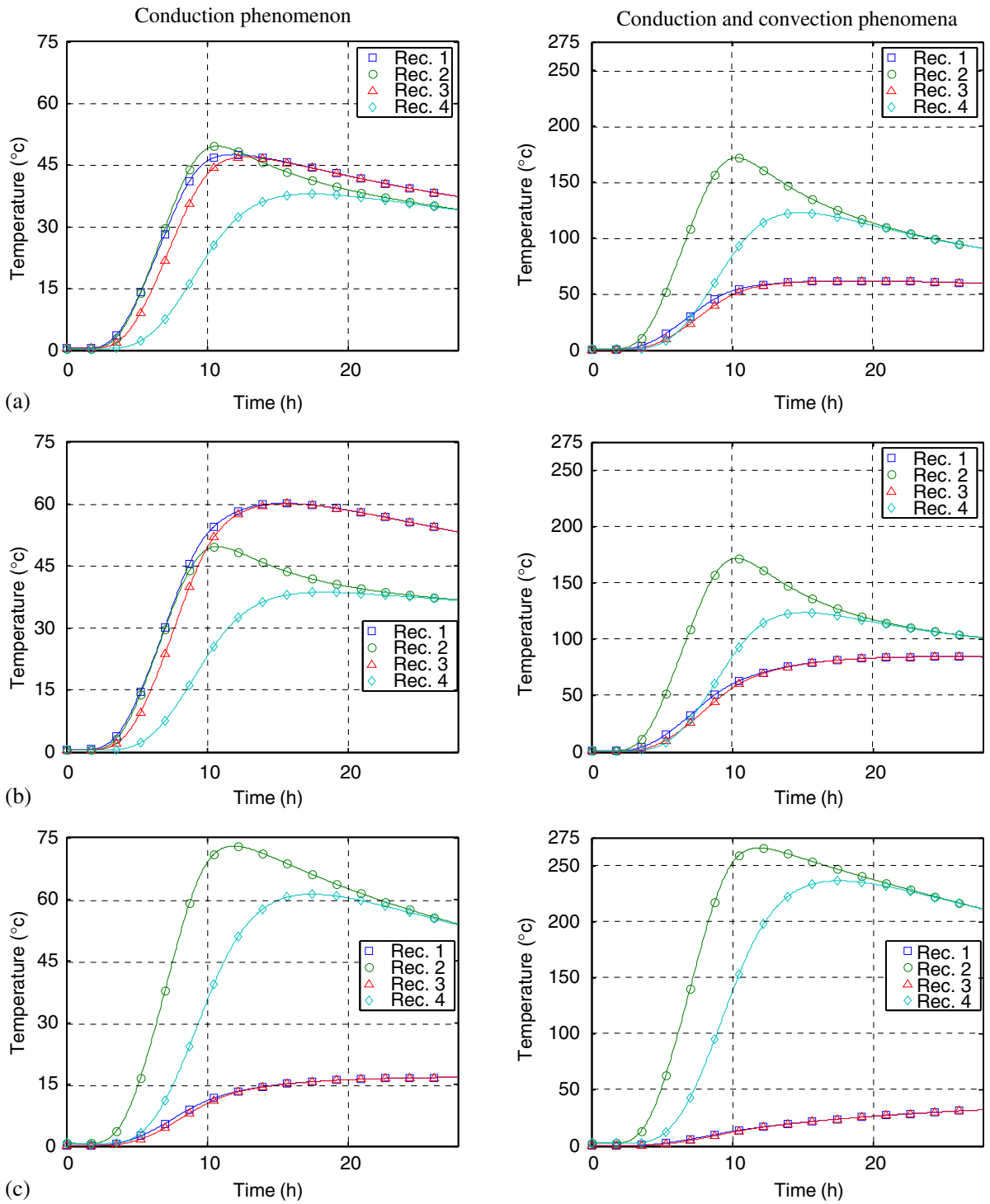

Fig. 9. Temperature curves for conduction alone and when both the conduction and convection phenomena are modelled, for receivers Rec. 1 to Rec. 4, for the three models, when the fluid is assumed to be water: (a) Model 1; (b) Model 2 and (c) Model 3. 
the results of this last case with those presented in Fig. 7(a) and (b), it can be seen that much higher temperatures are registered at the receivers placed in the air medium. This effect is in accordance with the fact that air allows a much higher diffusivity coefficient than water. As time advances $(t=10 \mathrm{~h})$, the energy is still propagating away from the source and a temperature rise has been registered at the layers below the sources' layer. However, it is interesting to note that the temperature has fallen very quickly in the air medium, confirming its higher diffusivity.

To better illustrate the temperature evolution over time, the temperature curves computed at receivers Rec. 1 to Rec. 4, are shown in Fig. 9. The first two receivers are placed abreast of the source power $(x=-0.45 \mathrm{~m})$ as shown in Fig. 6, while receivers Rec. 3 and Rec. 4, are located between heat sources $(x=-0.3 \mathrm{~m})$. In order to obtain more information about the system's behaviour, the receivers Rec. 1 and Rec. 3 are inside the concrete layer, while Recs. 2 and 4 are placed at the fluid medium close to the heat source layer.

The temperatures at receivers Rec. 1 are always higher than those found at Rec. 3, in the first part of the time window. Looking at the figures related to the conduction-convection heat transfer results, we can see much higher maximum amplitudes than for conduction alone. In the conduction-convection models, the receivers Rec. 2 and Rec. 3 reach maximum temperatures significantly higher than the other two receivers. This is because of the presence of the convection velocity in the fluid medium, which is a constant positive value in the $y$ direction. A similar behaviour is exhibited by Model 3 (see Fig. 9(c)) when only conduction is considered, because of the insulating layer position.

Notice that the results are linear in relation to the heat source power. Considering that each heat source only generates $100 \mathrm{~W}$ the maximum temperature reached by Model 3 would be $26.56{ }^{\circ} \mathrm{C}$, in spite of the $265.6{ }^{\circ} \mathrm{C}$ obtained for the present case.

The presence of an insulating layer at the bottom of the concrete layer and the absence of the convection phenomenon are responsible for the particular results registered in Fig. 9(b), where receiver Rec. 2 is not the one to record the maximum temperature, unlike the rest of temperature curves figures.

\section{Final remarks}

An approach for computing the transient heat transfer by conduction and convection across a layered medium has been described. This formulation proposes to calculate the heat responses first in the frequency domain. The methodology described requires the knowledge of fundamental solutions or Green's functions for a layered formation, and these were established using the continuity of temperatures and normal heat fluxes as boundary conditions at the media interfaces between the various layers. The heat field results for the multi-layer system are achieved considering the contribution of heat source (incident field) and heat surface terms (generated heat field). The verification of the mathematical formulation and the evaluation of the Green's functions' accuracy for the layered formation are given.

This technique seems to be suitable for studying a multi-layer floor construction subjected to multiple heat sources. The application results show that the presence and position of an insulating layer are important variables to be considered when studying radiant floor heating. The convective phenomenon has also been contemplated, representing an important contribution for the response.

\section{References}

[1] Özisik MN. Heat conduction, second ed. New York: Wiley; 1993.

[2] Monte F. Transient heat conduction in one-dimensional composite slab. A "natural" analytic approach. International Journal of Heat and Mass Transfer 2000;43:3607-19.

[3] Wang S, Chen Y. Transient heat flow calculation for multilayer constructions using frequency-domain regression method. Building and Environment 2003;38:45-61.

[4] Haji-Sheikh A, Beck JV. Temperature solution in multi-dimensional multi-layer bodies. International Journal of Heat and Mass Transfer 2002;45:1865-77.

[5] Sun Y, Wichman IS. On transient heat conduction in a onedimensional composite slab. International Journal of Heat and Mass Transfer 2004;47:1555-9.

[6] Chang YP, Kang CS, Chen DJ. The use of fundamental Green Functions for solution of problems of heat conduction in anisotropic media. International Journal of Heat and Mass Transfer 1973;16:1905-18.

[7] Shaw RP. An integral equation approach to diffusion. International Journal of Heat and Mass Transfer 1974;17:693-9.

[8] Divo E, Kassab A. A generalized BEM for steady and transient heat conduction in media with spatially varying thermal conductivity. In: Golberg MA, editor. Advances in boundary elements numerical and mathematical aspects. Boston: Computational Mechanics Publication; 1998. p. 37-76 (Chapter 2).

[9] Rizzo FJ, Shippy DJ. A method of solution for certain problems of transient heat conduction. AIAA Journal 1970;8: 2004-9.

[10] Sutradhar A, Paulino GH, Gray LJ. Transient heat conduction in homogeneous and non-homogeneous materials by the Laplace transform Galerkin Boundary Element Method. Engineering Analysis with Boundary Elements 2002;26(2):119-32.

[11] Stehfest H. Algorithm 368: numerical inversion of Laplace transform. Communications of the Association for Computing Machinery 1970;13(1):47-9.

[12] Davies MG. Wall transient heat flow using time-domain analysis. Building and Environment 1997;32(5):427-776.

[13] Chen Y, Wang S, Zuo Z. An approach to calculate transient heat flow through multilayer spherical structures. International Journal of Thermal Sciences 2003;42:805-12.

[14] Tadeu A, António J, Simões N. 25D Green's functions in the frequency domain for heat conduction problems in unbounded, half-space, slab and layered media. Computer Modeling in Engineering \& Sciences-CME 2004;6(1):43-58. 
[15] Carslaw HS, Jaeger JC. Conduction of heat in solids, second ed. Oxford: Oxford University Press; 1959.

[16] Ochiai Y. Steady heat conduction analysis in orthotropic bodies by triple-reciprocity BEM. Journal of Computer Modeling in Engineering and Sciences, CMES 2001;2(4):435-46.

[17] Simões N, Tadeu A, Godinho L. Transient heat transfer across a multi-layer wall. International Journal for Housing Science and its Applications 2003;27:287-300.
[18] Tadeu A, Kausel E. Green's Functions for two-and-a-half dimensional elastodynamic problems. Journal of Engineering Mechanics-ASCE 2000;126(10):1093-7.

[19] Bouchon M, Aki K. Discrete wave-number representation of seismic-source wave field. Bulletin of the Seismological Society of America 1977;67:259-77.

[20] Banerjee PK. The boundary element methods in engineering. England: McGraw-Hill; 1981. 